\title{
Crystal Growth, Optical and Dielectric Properties of L-Histidine Hydrochloride Monohydrate Nonlinear Optical Single Crystal
}

\author{
P. Koteeswari ${ }^{1}$, S. Suresh ${ }^{2 *}$, P. Mani ${ }^{1}$ \\ ${ }^{1}$ Department of Physics, Hindustan Institute of Technology, Padur, India \\ ${ }^{2}$ Department of Physics, Loyola College, Chennai, India \\ Email: "sureshsagadevan@yahoo.co.in
}

Received April 25, 2012; revised June 10, 2012; accepted July 1, 2012

\begin{abstract}
Optically transparent and bulk single crystal of 1-histidine hydrochloride monohydrate (LHHM) was successfully grown by slow evaporation technique. The cell parameters and the crystallinity of the grown crystal were estimated by the single crystal XRD. Optical transmittance of the crystal was recorded using the UV-vis-NIR spectrophotometer. The optical band gap and optical constant of the material were determined by using transmission spectrum. The dielectric loss and dielectric constant measurements as a function of frequency and temperature were measured for the grown crystal.
\end{abstract}

Keywords: Single Crystal; Slow Evaporation Technique; XRD; UV and Dielectric Studies

\section{Introduction}

The organic NLO materials are attracted by many scientists due to their frequency conversion efficiency, piezoelectric, pyro-electric properties and their wide applications in the recent technologies like lasers, optical communications and data storage [1]. New materials with high optical nonlinearities are quite important due to their extensive application in harmonic generation, amplitude and phase modulation, switching and other signal processing device [2-4]. The main goal to design the molecules with the third order nonlinearities is to incorporate them into the devices used in all types of optical signal processing [5-6]. Nonlinear optical (NLO) materials have shown potential application in optical information storage, optical logic gates, laser radiation protection and phase locked laser mode. Hence the interest in searching for NLO materials has increased gradually [7]. In addition to that organic molecules also have a great attention owing to their potential application in the frontier areas such as nonlinear optics (NLO), optical switching and light emitting diodes. Thus, the potential use of organic device materials in optoelectronics has now become a serious matter [8]. The present investigation is aimed at the growth of bulk LHHM single crystal by slow evaporation technique. The grown crystal has been subjected to single X-ray diffraction analysis, UV-vis transmission spectral analysis, optical band gap meas-

"Corresponding author. urements, and dielectric studies.

\section{Experimental Procedure}

The 1-histidine and hydrochloric acid were taken in equi molar ratio in double distilled water to prepare the saturated solution of 1-histidine hydrochloride monohydrate (LHHM). The solution obtained is stirred well at room temperature using a temperature controlled magnetic stirrer to yield a homogenous mixture of solution. Then the solution is filtered using a Whatmann filter paper and was allowed to evaporate at room temperature. The solution is recrystallized several times in order to increase the purity of the crystal. Optically clear and good quality seed crystal is kept inside the purified saturated solution and the solution is allowed to evaporate at room temperature, which produces an improved optically high quality within a period of 30 days. The photograph of the as grown single crystal is shown in Figure 1.

\section{Results and Discussion}

\subsection{Single Crystal X-Ray Diffraction}

Single crystal X-ray diffraction analysis was carried out to determine the lattice parameters. The grown crystals have orthorhombic structure with $P 2_{1} 2_{1} 2_{1}$ space group. The lattice parameter values of the grown crystals are $a=$ $6.82 \AA, b=8.91 \AA, c=15.286 \AA$. The single crystal data are in good agreement with reported values [9]. 


\subsection{Optical Transmittance Spectrum Study}

The optical transmission spectrum of LHHM single crystal was recorded in the wavelength region $300-900 \mathrm{~nm}$ and is shown in Figure 2. For optical fabrications, the crystal should be highly transparent in the considered region of wavelength [10] and [11]. Favorable transmittance of the crystal in the entire visible region suggests its suitability for second harmonic generation [12]. The UV absorption edge for the grown crystal was observed to be around $260 \mathrm{~nm}$. The dependence of optical absorption coefficient on photon energy helps to study the band structure and type of transition of electrons [13].

Optical absorption coefficient $(\alpha)$ was calculated from transmittance using the following relation:

$$
\alpha=\frac{1}{d} \log \left(\frac{1}{T}\right)
$$

where $T$ is the transmittance and $t$ the thickness of the crystal. As a direct band gap material, the crystal under study has an absorption coefficient $(\alpha)$ obeying the following relation for high photon energies $(h v)$.

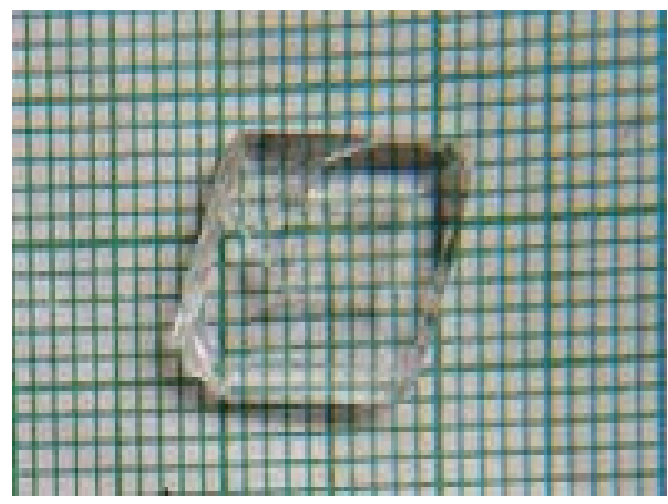

Figure 1. Photograph of as grown crystal.

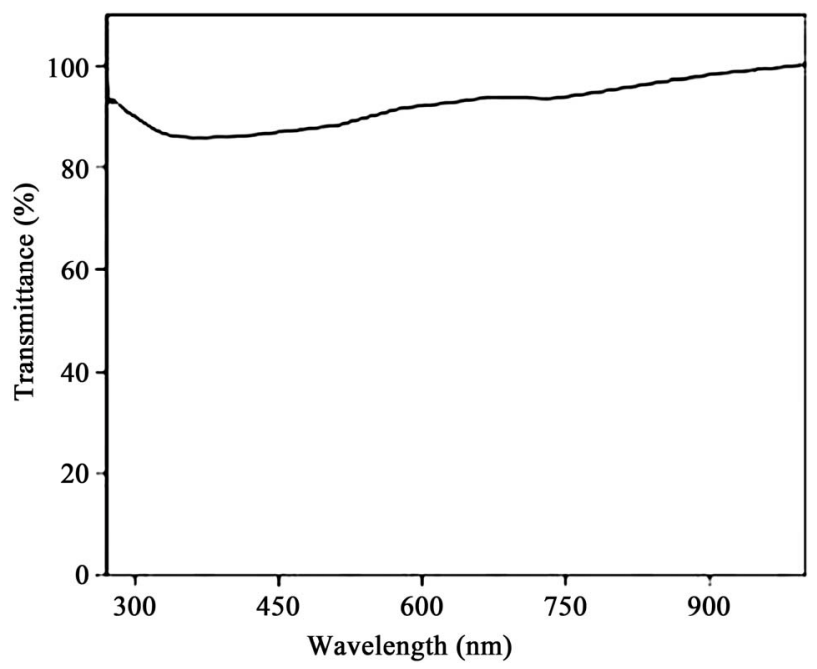

Figure 2. Transmission spectrum of the grown crystal (LHHM).

$$
\alpha=\frac{A\left(h v-E_{g}\right)^{1 / 2}}{h v}
$$

where $E_{g}$ is the optical band gap of the crystal and $A$ is a constant. A plot of variation of $(\alpha h v)^{2}$ versus $h v$ is shown in Figure 3. $E_{g}$ is evaluated using extrapolation of the linear part [14]. The energy absorption gap is of direct type and the band gap energy is found to be $3.90 \mathrm{eV}$.

\subsection{Determination of Optical Constant}

The dependence of optical absorption co-efficient with photon energy helps to study the band structure and the type of transition of the electron. The absorption coefficient $(\alpha)$ and the optical constant $(n, k)$ are determined from the transmission $(T)$ and reflection $(R)$ spectrum based on the following relations, $[15,16]$.

$$
T=\frac{(1-R)^{2} \exp (-\alpha t)}{1-R^{2} \exp (-2 \alpha t)}
$$

Reflectance can also be written in terms of absorption coefficient and from the $a b$

$$
R=\frac{1 \pm \sqrt{1-\exp (-\alpha t)+\exp (\alpha t)}}{1+\exp (-\alpha t)}
$$

and from the above equation, refractive index $(n)$ can also be derived as

$$
n=-\frac{(R+1) \pm \sqrt{3 R^{2}+10 R-3}}{2(R-1)}
$$

Figure 4 show the variation of refractive index $(n)$ as a function of wavelength $(\lambda)$, respectively. From the graphs, it is clear that refractive index $(n)$ depend on wavelength $(\lambda)$.

\subsection{Dielectric Studies}

The dielectric characteristics of the material are important

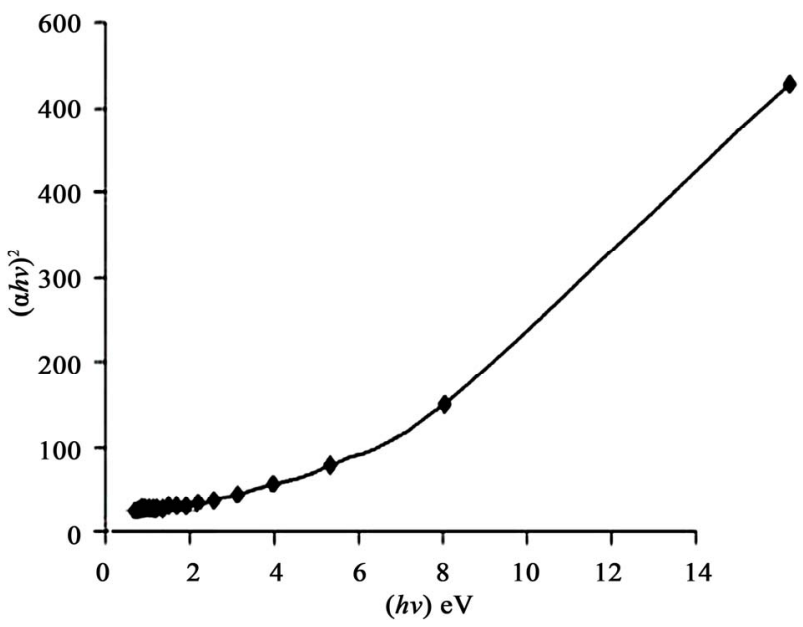

Figure 3. Plot of $(\alpha h v)^{2}$ vs photon energy of the title crystal. 
to study the lattice dynamics in the crystal. Hence, the grown crystal was subjected to dielectric studies using a HIOKI HITESTER model 3532-50 LCR meter in the frequency range from $50 \mathrm{~Hz}$ to $5 \mathrm{MHz}$ for different temperatures. The surface of the sample was electrode with silver paste for electrical contact. Figure 5 shows the plot of dielectric constant $\left(\varepsilon_{r}\right)$ versus log frequency. The dielectric constant has high values in the lower frequency region and then it decreases with the increase in frequency. The very high value of $\varepsilon_{r}$ at low frequencies may be due to the presence of all the four polarizations, namely, space charge, orientational, electronic and ionic polarization and its low value at higher frequencies may be due to the loss of significance of these polarizations gradually. From the plot, it is also observed that dielectric constant increases with an increase in temperature, and this is attributed due to the presence of space charge polarization near the grain boundary interfaces, which depends on the purity and perfection of the sample [17].

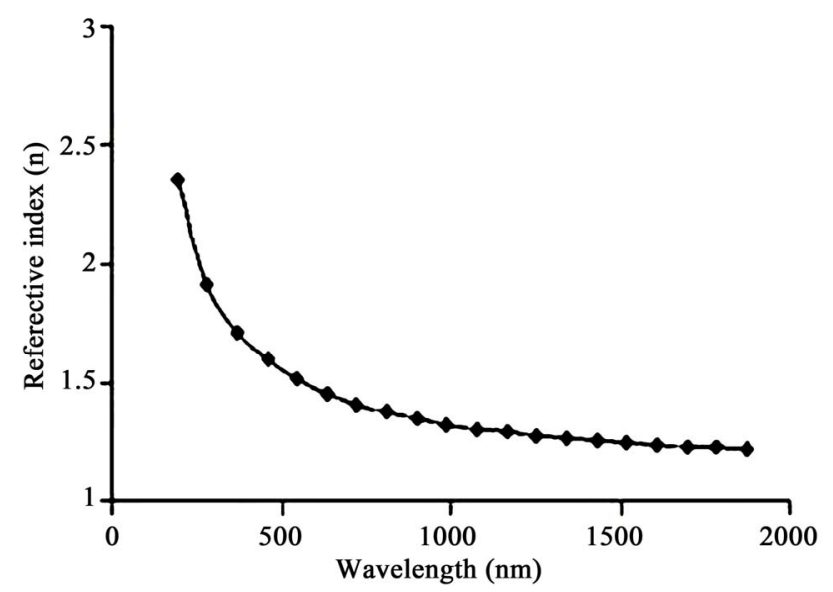

Figure 4. Variation of refractive index with wavelength.

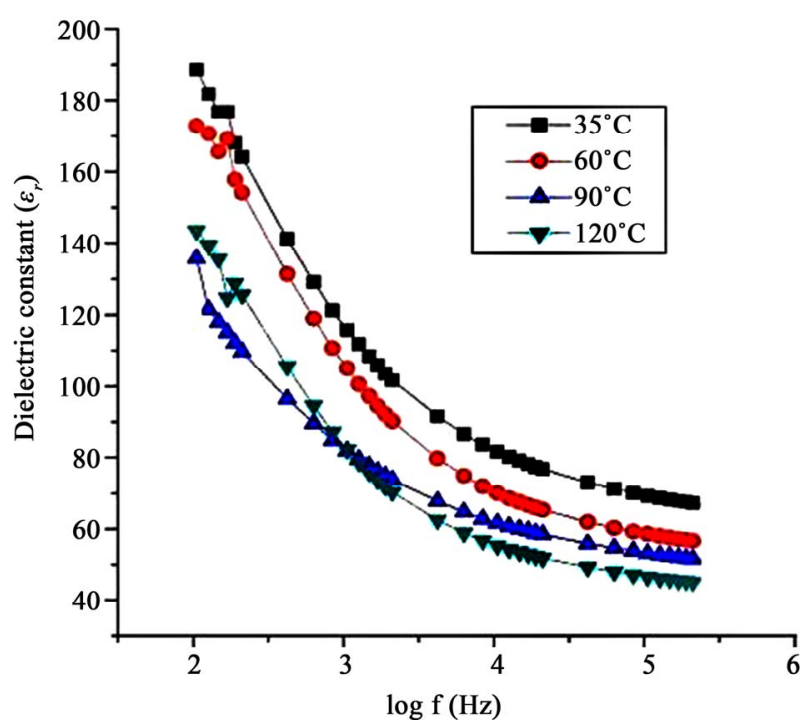

Figure 5. Variation of dielectric constant with log frequency.

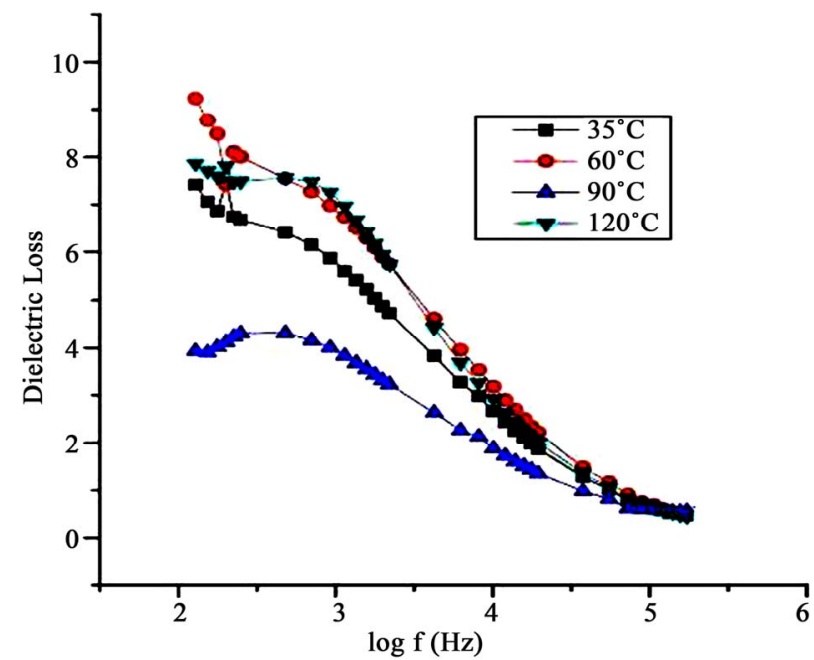

Figure 6. Variation of dielectric loss with log frequency.

The variation of dielectric loss with frequency is shown in Figure 6. The characteristics of low dielectric loss with high frequency for the sample suggest that it possesses enhanced optical quality with lesser defects and this parameter is of vital importance for nonlinear optical applications [18].

\section{Conclusion}

Bulk single crystal of semi-organic LHHM was grown from aqueous solution by a slow evaporation technique. Single crystal X-ray diffraction studies confirm that the grown crystal belongs to orthorhombic crystal system with space group $P 2_{1} 2_{1} 2_{1}$. The optical transmission analysis indicates that LHHM has a wide transparency window in the entire visible and near IR regions with a lower cutoff wavelength at $260 \mathrm{~nm}$. The band gap was estimated to be $3.90 \mathrm{eV}$. The variation of dielectric constant $\left(\varepsilon^{\prime}\right)$, dielectric loss and imaginary dielectric constant $\left(\varepsilon^{\prime \prime}\right)$ were studied as a function of frequency at different temperatures.

\section{REFERENCES}

[1] N. B. Singh, T. Henningsen, E. P. A. Metz, R. Hamacher, E. Cumberledge, R. H. Hopkins and R. Mazelsky, "Solution Growth of Vanillin Single Crystals," Materials Letters, Vol. 12, No. 4, 1991, pp. 270-275. doi:10.1016/0167-577X(91)90012-U

[2] E. W. Van Stryland, H. Vanherzeele, M. A. Woodall, M. J. Soileau, A. L. Smirl, S. Guha and T. F. Bogess, "Two Photon Absorption, Nonlinear Refraction, and Optical Limiting in Semiconductors," Optical Engineering, Vol. 24, 1985, p. 613.

[3] T. Wei, D. J. Hagan, E. W. Van Stryland, "Sensitive Measurement of Optical Nonlinearities Using a Single Beam," IEEE Journal of Quantum Electronics, Vol. 26, No. 4, 1990, pp. 760-769. 
[4] J. J. Rodrigues Jr., L. Misoguti, F. D. C. R. Nunes, C. R. Mendonca and S. C. Zilio, "Optical Properties of LThreonine Crystals," Optical Materials, Vol. 22, No. 3, 2003, pp. 235-240. doi:10.1016/S0925-3467(02)00270-7

[5] M. Somac, A. Somac, B. L. Davies, M. G. Humphery and M. S. Wong, "Third-Order Optical Nonlinearities of Oligomers, Dendrimers and Polymers Derived from Solution Z-Scan Studies," Optical Materials, Vol. 21, No. 1-3, 2002, pp.485-488.

[6] L. V. Natarajan, R. L. Sutherland, V. P. Tondiaglia, T. J. Bunning, W. W. Adams and J. Nonlinear, "Electro-Optical Switching Characteristics of Volume Holograms in Polymer Dispersed Liquid Crystals," Optical Physics and Materials, Vol. 5, No. 1, 1996, pp. 89-98.

[7] X. Xu, W. Qiu, Q. Zhou, J. Tang, F. Yang, Z. Sun and P. Audebert, "Nonlinear Optical Absorption Properties of Two Multisubstituted p-Dimethylaminophenylethenyl Pyridiniums," Journal of Physical Chemistry B, Vol. 112, No. 16, 2008, pp. 4913-4917. doi:10.1021/jp7103775

[8] J. G. Breitzar, D. D. Dlott, L. K. Iwaki, S. M. Kirkpatrick and T. B. Rauchturs, "Third-Order Nonlinear Optical Properties of Sulfur-Rich Compounds," Journal of Physical Chemistry A, Vol. 103, No. 35, 1999, pp. 6930-6937. doi:10.1021/jp990137f

[9] J. Madhavan, S. Aruna, P. C. Thomas, M. Vimalan, S. A. Rajasekar and P. Sagayaraj, "Growth and Characterization of L-Histidine Hydrochloride Monohydrate Single Crystals," Crystal Research and Technology, Vol. 42, No. 1, 2007, pp. 59-64. doi:10.1002/crat.200610771

[10] V. Krishnakumar and R. Nagalakshmi, "Crystal Growth and Vibrational Spectroscopic Studies of the Semiorganic Non-Linear Optical Crystal-Bisthiourea Zinc Chloride," Spectrochim Acta Part A: Molecular and Biomolecular Spectroscopy, Vol. 61, No. 3, 2005, pp. 499-507. doi:10.1016/j.saa.2004.04.014

[11] V. Krishnakumar and R. J. Xavier, "FT Raman and FT-IR
Spectral Studies of 3-Mercapto-1,2,4-Triazole," Spectrochim Acta Part A: Molecular and Biomolecular Spectroscopy, Vol. 60, No. 3, 2004, pp. 709-714. doi:10.1016/S1386-1425(03)00281-6

[12] S. A. Roshan, J. Cyriac and M. A. Ittyachen, "Growth and Characterization of a New Metal-Organic Crystal: Potassium Thiourea Bromide," Materials Letters, Vol. 49, No. 5, 2001, pp. 299-302. doi:10.1016/S0167-577X(00)00388-8

[13] N. Tigau, V. Ciupinaa, G. Prodana, G. I. Rusub, C. Gheorghies and E. Vasilec, "Influence of Thermal Annealing in Air on the Structural and Optical Properties of Amorphous Antimony Trisulfide Thin Films," Journal of Optoelectronics and Advanced Materials, Vol. 6, No. 1, 2004, pp. 211-217.

[14] A. K. Chawla, D. Kaur and R. Chandra, "Structural and Optical Characterization of $\mathrm{ZnO}$ Nanocrystalline Films Deposited by Sputtering," Optical Materials, Vol. 29, No. 8, 2007, pp. 995-998. doi:10.1016/j.optmat.2006.02.020

[15] B. L. Zhu, C. S. Xie, D. W. Zeng, W. L. Song and A. H. Wang, "Investigation of Gas Sensitivity of Sb-Doped ZnO Nanoparticles," Materials Chemistry and Physics, Vol. 89, No. 1, 2005, pp. 148-153. doi:10.1016/j.matchemphys.2004.08.028

[16] A. Ashour, N. El-Kadry and S. A. Mahmoud, "On the Electrical and Optical Properties of CdS Films Thermally Deposited by a Modified Source," Thin Solid Films, Vol. 269, No. 1-2, 1995, pp. 117-120. doi:10.1016/0040-6090(95)06868-6

[17] C. P. Smyth, "Dielectric Behaviour and Structure," McGrawHill, NewYork, 1965.

[18] C. Balarew and R. Duhlew, "Application of the Hard and Soft Acids and Bases Concept to Explain Ligand Coordination in Double Salt Structures," Journal of Solid State Chemistry, Vol. 55, No. 1, 1984, pp. 1-6. doi:10.1016/0022-4596(84)90240-8 